\title{
Concept System and Function of Dynamic Symmetry in Mechanical Product Structure
}

\author{
Fan Liu and Zhiyong Ma*
}

\author{
Faculty of Mechanical Engineering and Mechanics, Zhejiang Provincial Key Lab of Part Rolling Technology, Ningbo \\ University, Ningbo, 315211, China
}

\begin{abstract}
Dynamic symmetry is a common phenomenon in mechanical product structures, and is used for describing the regular motion process over time in mechanical systems. It plays an important role in implementing product functions, transfering and transforming movement motion, increasing the strength of product functions, expanding the scope of the functions. For the case that the lack of research about dynamic symmetry and imperfection of symmetric system, based on the analysis of instances, a new concept system of mechanical dynamic structure symmetry was established by taking the different properties of the geometric character and the time character of the motion process as standards. The concept system is made up of rotation dynamic symmetry, translation dynamic symmetry, scaling dynamic symmetry, combinatorial dynamic symmetry, time-translation dynamic symmetry and time-inversion dynamic symmetry. Among the concept systemthe author has a symmetry theory analysis of many instances to verify the validity and rationality of the established symmetry concept system. Finally, the functions of dynamic symmetry in realizing the function of transferring or transforming motion, matching the space-time characters and improving the performance of mechanical product functions was proposed. The concept system can completely describes the existences of dynamic structure symmetries in mechanical systems, and can offer an academic basis for further research on the functions and application laws of dynamic structure symmetry in mechanical systems.
\end{abstract}

Keywords: Concept system, dynamic structure symmetry, function, geometric character of motion, product structure, time character of motion.

\section{INTRODUCTION}

Symmetry is a common phenomenon in the real world. For example, the structure of crystals, the shape of vertebrates, the artificial products such as autocars and airplanes all have symmetry. In many domains, such as physics, biology, mathematics and philosophy, many people have researched the theory of symmetry for a long time and achieved a lot. Especially in physics, a complete concept system of symmetry was established [1] and many important principles and laws about symmetry were proposed. In 1894, Pierre Curie had brought out the principle of symmetry [2]. Thereafter, Albert Einstein proposed the important functions of symmetry in the research of physics [3]. Until now, the principle of symmetry is still a most fundamental principle and it still directs the research of physics $[4,5]$.

In mechanical systems, a mechanical product realizes its functions through the motions and interactions, which must observe certain physical principles. There are many closed relationships between physical principles and symmetries. For example, the law of conservation of momentum can be deduced from space translation symmetry, and various space-time symmetries also can be deduced from various conservation laws [6]. In a kind of universal joint for autocar showed in Fig. (1) [7], the structure 1 and structure 2 both have static rotation symmetry. By working with the ball 3, they can realize the function of universally transferring rotation motion. In a kind of hand pump showed in Fig. (2) [8], the component 1 and 2 both have sway dynamic symmetry, the component 4 has translation dynamic symmetry. The component 1 dose sway motion and drives the component 4 to do vertical reciprocating rectilinear motion, so the function of getting water can be realized.

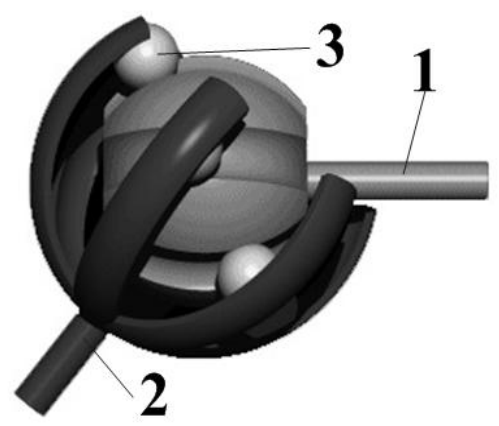

Fig. (1). An universal joint for autocar [7].

Symmetry also widely exists in mechanical product structure. Concept systems of mechanical static structure symmetry have been proposed by Barrenscheen and Z. Y. Ma $[9,10]$. Partial functions of static structure symmetry in the design, manufacture and assembly of mechanical product have be proposed by P. L. Prasad, Susan J. T. and so on [11, 
12]. Until now, there still has not any systematic researches on mechanical dynamic structure symmetry. Based on analysis of a lot of instances, this article established a concept system of mechanical dynamic structure symmetry, and proposed some functions of dynamic symmetry in realizing the functions of mechanical products. The research results of this article are the academic basis of further researches on the functions and application laws of mechanical dynamic structure symmetry.

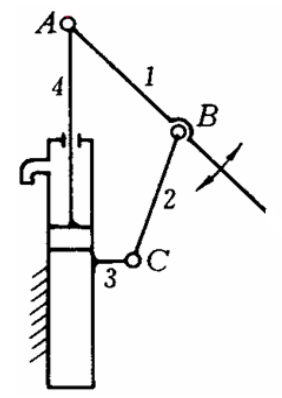

Fig. (2). A hand pump [8].

\section{DEFINITION OF MECHANICAL DYNAMIC STRUCTURE SYMMETRY}

In the macroscopical symmetry of physics, space symmetry is a kind of static symmetry while time symmetry is a kind of dynamic symmetry. The mechanical dynamic structure symmetry is defined as following: if one or more structures have a regular motion process over time in a mechanical system, then this mechanical system has dynamic symmetry. Dynamic structure symmetry has three key properties: symmetry main body, symmetry benchmark and symmetry component [10]. The symmetry main body is the geometrical character of the motion process of the mechanical structure, the symmetry component is the structure which has a regular motion process, the symmetry benchmark is time. In a kind of punch showed in Fig. (7), the diamond plate 1 is the driving part, which does rotation motion and drives the shift fork 3 by the slide block 2 fixed on the diamond plate. The disc 4 also does rotation motion and it drivers punch pin 6 to do vertical reciprocating rectilinear motion. In the punch, the structures from 1 to 6 all have dynamic structure symmetry because they all have a regular motion process. For example, the motion process of diamond plate 1 is a unidirectional entire rotation, and the motion process of the punch pin 6 is a reciprocating rectilinear motion. The dynamic symmetries of every structure in the punch are showed in Table $\mathbf{1}$.

\section{CLASSIFICATION SYSTEM OF MECHANICAL DYNAMIC STRUCTURE SYMMETRY}

Dynamic structure symmetry has various existing forms in mechanical product structures, which can be described as different properties of symmetry main body and symmetry

Table 1. The dynamic symmetries of every structure in the punch.

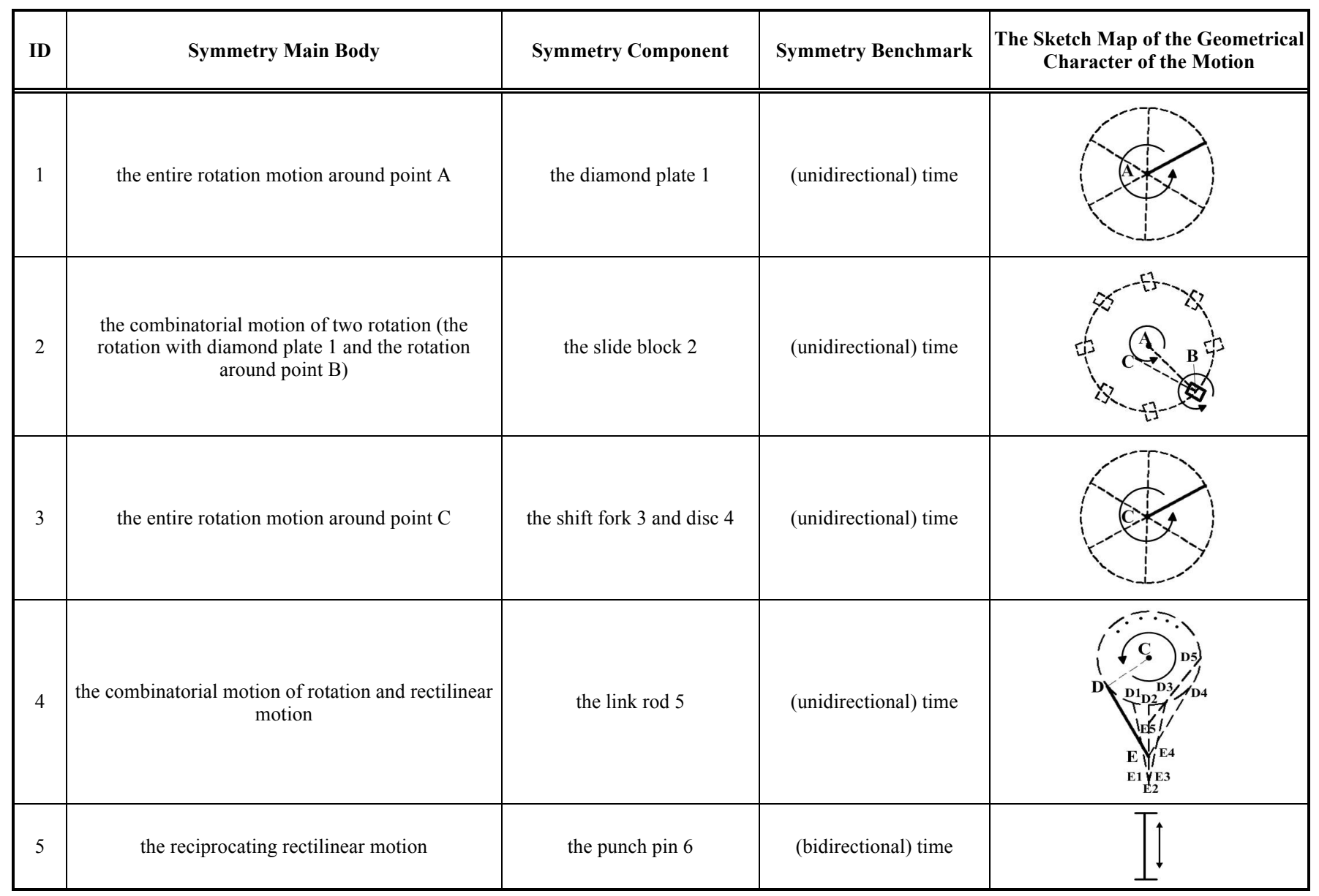


benchmark. The symmetry main body describes the geometrical character of the motion process of dynamic structure symmetry, and the symmetry benchmark describes the time character of the motion process. According to the different properties of symmetry main body and symmetry benchmark, the classification system of mechanical dynamic structure symmetry was established, showed in Fig. (3).

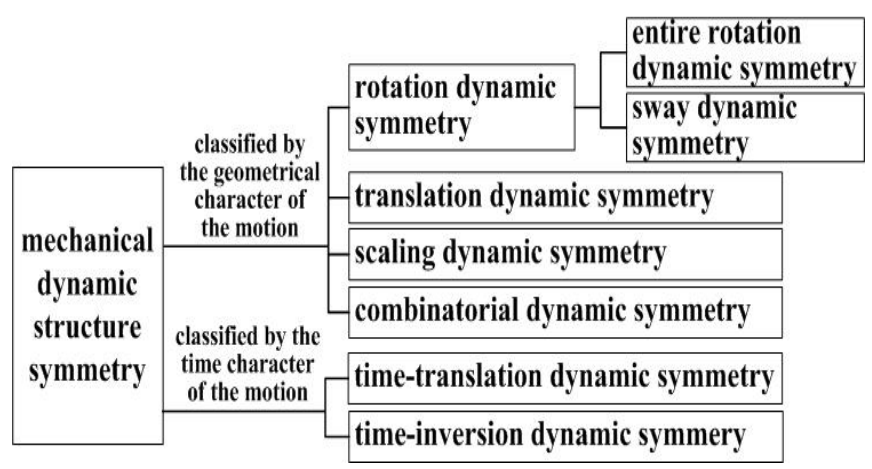

Fig. (3). The classification system of mechanical dynamic structure symmetry.

\subsection{The Classification System Classified by the Geometrical Character of the Motion Process}

According to the types of geometrical character of the motion process, dynamic structure symmetry can be classified as rotation dynamic symmetry, translation dynamic symmetry, scaling dynamic symmetry and combinatorial dynamic symmetry.

If a structure does a regular rotation motion, then it has rotation dynamic symmetry. According to the integrality of the rotation, rotation dynamic symmetry can be classified as entire rotation dynamic symmetry and sway dynamic symmetry. In a valve mechanism of engine showed in Fig. (4) [8], the cam 1 does rotation motion and the cam carrier 2 does reciprocating sway motion, so the cam 1 has entire rotation dynamic symmetry and the cam carrier 2 has sway dynamic symmetry.

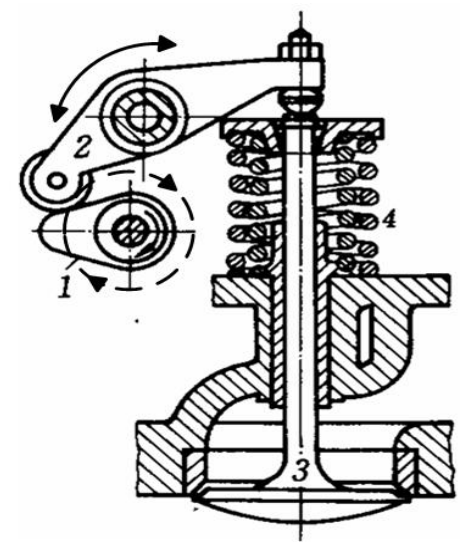

Fig. (4). A valve mechanism of engine [8].

If a structure does regular rectilinear motion, then it has translation dynamic symmetry. In a knife feed mechanism of machine tool showed in Fig. (5) [8], the cam 1 drives the cam carrier 2 does the reciprocating sway motion around point $\mathrm{O}$, then the knife rest 4 does horizontal reciprocating rectilinear motion drove by the gear and rack mechanism to realize the function of engage and retract. So the knife rest 4 has translation dynamic symmetry, and the cam carrier 2 has sway dynamic symmetry. The punch pin 6 showed in Fig. (7) also has translation dynamic symmetry.

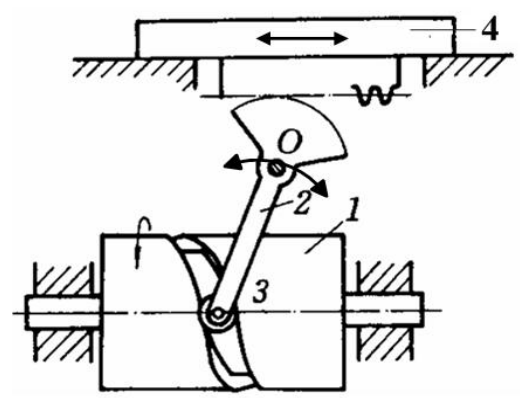

Fig. (5). A knife feed mechanism of machine tool [8].

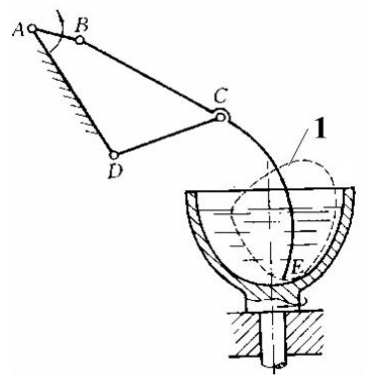

Fig. (6). The sketch map of an agitator [8].

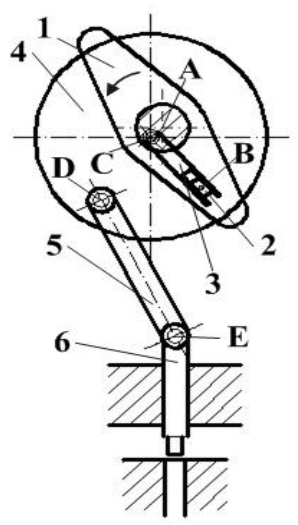

Fig. (7). The sketch map of a punch.

If the motion track of a structure is a curve with a certain rule, then the structure has scaling dynamic symmetry. In an agitator showed in Fig. (6) [8], the motion track of the link rod 2 is a regular closed curve (the curve 1), so it has scaling dynamic symmetry.

If a structure does two or more basic motions at one time, then it has combinatorial dynamic symmetry. For example, the slide block 2 showed in Fig. (7) does rotation motion around point $\mathrm{A}$, at the same time, it also rotates around point $\mathrm{B}$, so it has combinatorial dynamic symmetry.

\subsection{The Classification System Classified by the Time Character of the Motion Process}

According to the time character of the motion process, dynamic structure symmetry can be classified as timetranslation dynamic symmetry and time-inversion dynamic symmetry. If one or more structures have a regular motion 
process over time, and the motion process will remain unchanged when the time parameter $t$ is replaced by $-t$, then this mechanical system has time-inversion dynamic symmetry, otherwise, it has time-translation dynamic symmetry. In a punch showed in Fig. (7), if the direction of the rotation motion of the diamond plate 1 is changed from counter-clockwise to clockwise, then the direction of the motion of the slide block 2, the shift fork 3, disc 4 and the link rod 5 will all changed contrarily, so they all have timetranslation dynamic symmetry; but the motion of the punch pin 6 is still a reciprocating rectilinear motion, so it has timeinversion dynamic symmetry.

\section{FUNCTION OF DYNAMIC STRUCTURE SYMMETRY IN MECHANICAL SYSTEMS}

Symmetry widely exists in mechanical systems, and it has closed relationships with the motion process, the functions and the realization principles of mechanical systems. So it can systematically direct the designers to apply symmetry to achieve the design demands of mechanical products and offer a new method to assist product design. This article partially describes the functions of dynamic symmetry in realizing the function of transferring or transforming motion, matching the space-time characters and improving the performance of mechanical product functions.

\subsection{Function of Dynamic Structure Symmetry in Realizing the Function of Transferring or Transforming Motion}

It is one of the most common functions of dynamic structure symmetry that realize the function of transferring or transforming motion. If two or more structures are connected by kinematic pairs, and a dynamic structure symmetry belonging to a structure can drive another dynamic structure symmetry belonging to another structure, then the function of transferring or transforming motion can be realized. By changing the sizes of structures and the types of kinematic pairs, it can export different motions according to a certain input motion. In a lift mechanism showed in Fig. (8) [8], the crank $\mathrm{AB}$ and $\mathrm{CD}$ both have entire rotation dynamic symmetry, the link rod $\mathrm{BC}$ has translation dynamic symmetry. By setting the lengths of crank AB and CD to be same, it can transform the rotation motion of crank $A B$ to the vertical rectilinear motion of link rod BC. So the function of rising and falling the lift table can be realized, and the lift table can be always kept horizontal.

\subsection{Function of Dynamic Structure Symmetry in Matching the Space-Time Characters of Product Functions}

In mechanical structure design, many dynamic structure symmetries are adopted to realize the functions of mechanical products. By designing the two main key elements of the motion process felicitously, geometrical character and the time character, the dynamic structure symmetry can realize the space-time characters of product functions. If product functions or its subfunctions are arranged regularly in space, or its space locations are changed regularly over time, the product functions can be realized by dynamic structure symmetry. Generally speaking, the geometrical character of the motion process of dynamic structure symmetry is accordant with the space distribution of the product function, and the time character of the motion process is accordant with the time benchmark of the product function.

\subsection{Function of Dynamic Structure Symmetry in Improving the Performance of Product Functions}

By adding a structural component which has dynamic structure symmetry into a mechanism, the working space range, working time range and the effect intensity of product functions can be improved. In a steel stock push mechanism showed in Fig. (9) [8], two link rod 2 and 3 which both have dynamic structure symmetry were added into a four-bar slider-crank mechanism, then the displacement of slider 5 could be extended largely.

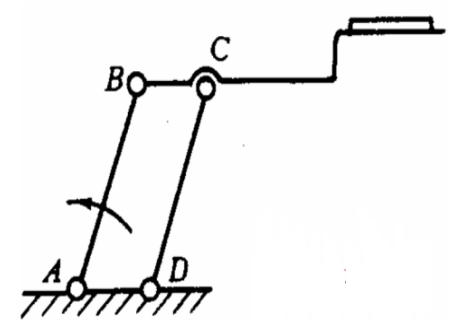

Fig. (8). A lift mechanism [8].

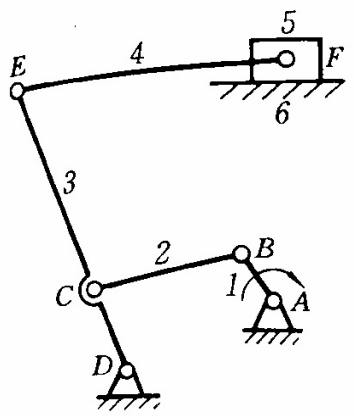

Fig. (9). A steel stock push mechanism [8].

\section{CONCLUSION}

Structure symmetry widely exists in mechanical products and plays an important role. Dynamic structure symmetry describes the laws of motion of mechanical product structures. Based on analysis of a lot of instances, a classification system of mechanical dynamic structure symmetry was established and the function of mechanical dynamic structure symmetry was researched. The research results of this article can offer an academic basis for further research on the functions and application laws of dynamic structure symmetry in mechanical systems.

\section{CONFLICT OF INTEREST}

The authors confirm that this article content has no conflict of interest. 


\section{ACKNOWLEDGEMENTS}

This work was supported by the National Science Foundation of China (No. 51305213), the Department of Education of Zhejiang Province of China (No. Y201224583) and the K.C. Wong Magna Fund in Ningbo University.

\section{REFERENCES}

[1] G. Darvas. "Generalisation of the concept of symmetry and its classification in physics", Acta Physica Hungarica A: Heavy Ion Physics, vol. 19, no. 3-4, pp. 373-379, 2004.

[2] K. Zhao. Semi-quantitative physics, High Education Press, Beijing, 1993.

[3] C. Yang. "Symmetry and Physics", Chi J. Nat, vol. 17, no. 5, pp. 247-256, 1995.

[4] K.A. Brading. "Which symmetry? Noether, Weyl, and conservation of electric charge", Stud. Hist. Philos. Mp., vol. 33, no. 1, pp. 3-22, 2002.

[5] M. Chaichian, A. Tureanu, G. Zet. "Twist as a symmetry principle and the non-commutative gauge theory formulation", Phy. Lett. B, vol. 651 , no. 4, pp. 319-323, 2007.
[6] Z. Yan. "The symmetry and law of conservation", J. Qiannan Normal College Nationalities, vol. 23, no. 3, pp. 13-16, 2003.

[7] Baidu Corporation. cardan joint, from http://baike.baidu.com/view/ 55664.htm.

[8] H. Sun, Z. Cheng, and W. Ge. Theory of machines and mechanisms, China Machine Press, Beijing, 2006.

[9] J. Barrenscheen. Die Systematische Ausnutzung von SymmetrieEigenschaften Beim Konstruieren, Department of Mechanical Engineering and Electrical Engineering, Technical University of Braunschweig, 1990

[10] Z. Ma, P. Feng, and Q. Qiu. "Concept system and application method of mechanical symmetry“. J. Zhejiang Univ. (Eng. Sci.), vol. 44, no. 12, p. 2354-2359, 2010.

[11] P. L. Prasad, R. Nandakumar and V. Ramamurti. "Estimation of machining errors using the concept of cyclic symmetry", Int. J. Mach. Tool Manu., vol. 37, no. 5, pp. 635-647, 1997.

[12] S.J. Tate, G.E.M. Jared, K.G. Swift. "Detection of symmetry and primary axes in support of proactive design for assembly", In: $5^{\text {th }}$ Symposium on Solid Modeling and Applications, Ann Arbor, pp. $151-158,1999$. 